http://www.jfas.info

\title{
APPLICATION OF METABOLITE PROFILING AND ANTIOXIDANT ACTIVITY IN ASSESSING THE QUALITY OF PROCESSED AND UNPROCESSED STINGLESS BEE'S PROPOLIS
}
A. Azemin ${ }^{1}$, N. B. Md-Zin ${ }^{1}$, M. M. Mohd-Rodi ${ }^{1}$, A. S. Kim-Chee ${ }^{1}$, A. J. Zakaria ${ }^{1,2}$ and K. S. Mohd $^{1, *}$

${ }^{1}$ Faculty of Bioresources and Food Industry, Universiti Sultan Zainal Abidin, 22200 Besut, Terengganu, Malaysia

${ }^{2}$ Centre for Farm Management, University Sultan Zainal Abidin, 22200 Besut, Terengganu, Malaysia

Published online: 08 August 2017

\begin{abstract}
This paper investigates the effect of processing and extraction method on chemical profiles and antioxidant activity of Malaysian stingless bee propolis. High Performance Thin Layer Chromatography (HPTLC) analysis in combination with chemometric shows that some of the compounds were degraded or not detected in processed sample, but the profiles were not affected by extraction method. Fourier Transform Infrared (FTIR) analysis shows that extraction methods significantly affect chemical profiles. Total phenolic content (TPC) and antioxidant activity were in agreement with HPTLC analysis, where unprocessed propolis possesses significantly higher TPC and antioxidant activity regardless of extraction method. This finding shows that metabolite profiling is a useful tool in assessing the quality of propolis.
\end{abstract}

Keywords: propolis; Heterotrigona itama; chemometric.

Author Correspondence, e-mail: khamsahsuryati@unisza.edu.my

doi: http://dx.doi.org/10.4314/jfas.v9i2s.40 


\section{INTRODUCTION}

Stingless bees generated apiculture products including honey, beebread (beehive pollen) and propolis. These products are well known for their nutritional and therapeutic properties. Among all, propolis stands out in term of biological activities. Propolis is referred to resinous material consists of $50 \%$ resins (composed of flavonoids and various phenolic acids), 30\% wax, $10 \%$ essential oils, $5 \%$ pollen and $5 \%$ various organic compounds [1] collected by stingless bees from leaf buds or plant nectar. Stingless bees produced propolis and use it for their construction material in order to seal the crack or the hole of the beehive, as well as for beehives protection from adverse weather conditions and hive infections [2]. Propolis has been used in traditional and folk medicine for centuries [3]. It has been reported to have many biological activities such as antioxidant [4], antibacterial, antifungal and antiviral [5], anti-inflammatory and wound healing [6] as well as antitumor upon human breast cancer [7]. As propolis is a potential therapeutic and health-promoting agent, the potential of commercialization exploitation is huge. Despite this, the processing method including method of extraction of raw material for getting the most benefit is found to be challenging.

Since propolis is a mixture of resin and wax, it is sometime hard and brittle that makes it difficult to handle and store in small container. In traditional practice, propolis was processed by heating in high temperature until propolis turn into soft and pliable material and be able to put in small containers and kept in $-20^{\circ} \mathrm{C}$ until further process. We hypothesized by heating the propolis in high temperature; some of the metabolites will be degraded or vanished all together. This process will diminished some of the important biological property as a result of metabolite degradation. In this work, we investigate the effect of processing method on the propolis from stingless bee species Heterotrigona itama. We also compared the type of extraction method in order to get the best quality of propolis extract. The quality of propolis was assessed through metabolite profiling namely FTIR and HPTLC coupled with chemometric analysis, antioxidant activity and total phenolic content.

\section{EXPERIMENTAL}

\subsection{Chemical and Reagents}


Methanol, ethyl acetate, acetic acid, toluene and dimethyl sulfoxide (DMSO) were purchased from Merck Sdn. Bhd. (Selangor, Malaysia); 2, 2- diphenyl-picryl-hidrazyl (DPPH), quercetin was acquired from Sigma-Aldrich (M) Sdn. Bhd. (Kuala Lumpur, Malaysia); Trolox ${ }^{\circledR}$ was purchased from Calbiochem ${ }^{\circledR}$; TLC silica gel 60 F254 glass plates was purchased from Merck Millipore (Darmstadt, Germany).

\subsection{Sample Preparation and Extraction}

In this work, processed and unprocessed propolis raw materials from Heterotrigona itama's stingless bee species were analyzed. Both processed and unprocessed were obtained from the local beekeeper. For processed sample, raw propolis was heated in high temperature for a period of time. Meanwhile, no treatment was applied to unprocessed sample as it was collected freshly from the hives. Both materials were extracted with ethanol by three methods of extraction: maceration, sonication and maceration-sonication. For maceration, both materials were macerated with ethanol for 3 days while for sonication, samples were sonicated at $37^{\circ} \mathrm{C}$ for 1 hour. As for maceration-sonication method, samples were macerated for 3 days and sonicated for 1 hour at $37^{\circ} \mathrm{C}$, consecutively. All extracts were then filtered, and were reduced under vacuum pressure at $45^{\circ} \mathrm{C}$. The crude extracts were stored in vials and kept in $4^{\circ} \mathrm{C}$ prior analysis. In this work, the propolis samples were labelled as P-M (Processed-Maceration), UP-M (UnProcessed-Maceration), P-S (Processed-Sonication), UP-S (UnProcessed-Sonication), P-MS (Processed-Maceration-Sonication) and UP-MS (UnProcessed-Maceration-Sonication).

\subsection{Fourier Transform Infrared Spectroscopy (FTIR) Analysis}

The FTIR spectra were obtained and recorded using IRPrestige-21 Shimadzu Fourier Transform Infrared Spectrophotometer (Tokyo, Japan) equipped with air-cooled ceramic infrared light source and DLATGS (Deuterated Triglycine Sulfate doped with L-Alanine) detector. The IR measurements were made at a resolution of $4 \mathrm{~cm}^{-1}$, and 16 inferograms were co-added before the Fourier transformation. The background spectra were recorded prior to analysis of the samples. The single-reflection Attenuated Total Reflectance (ATR) scan technique was used directly for all propolis samples. The sticky extracts of processed and unprocessed propolis samples were placed direct on the diamond prism for data acquisition. 
The data were recorded at the middle-IR range of $4000-400 \mathrm{~cm}^{-1}$.

\subsection{High Performance Thin Layer Chromatography (HPTLC) Analysis}

High Performance Thin Layer Chromatography analysis were carried out using HPTLC system equipped with CAMAG Automatic TLC Sampler 4 (ATS4, Muttenz, Switzerland) for sample application (which equipped with $25 \mu \mathrm{L}$ syringe), CAMAG TLC Scanner 4, Muttenz, Switzerland) for densitometry and spectra detection, CAMAG TLC Visualiser (DXA252 Digital Camera) for visualization and winCATS 1.4.9 software for data handling and processing. Development of plate was done manually using CAMAG ${ }^{\circledR}$ Twin Trough Chamber for $20 \times 10 \mathrm{~cm}$ plates with stainless steel lid. All ethanolic propolis extract samples were weighed up for $30 \mathrm{mg}$ and dissolved in $1 \mathrm{~mL}$ of methanol to get final concentration of 30 $\mathrm{mg} / \mathrm{mL}$. All samples prepared were sonicated for 30 minutes and centrifuged. The supernatant were spotted on TLC plates. The HPTLC analysis in this work was carried out on TLC precoated with silica gel $60 \mathrm{~F}_{254}$ glass plates, $20 \mathrm{~cm} \mathrm{x} 10 \mathrm{~cm}$, (layer thickness of $0.2 \mathrm{~mm}$ ) (Merck, Germany). Eight microliters of each propolis sample was streaked in the form of narrow bands with a length of $8.0 \mathrm{~mm}$ from the bottom edge, $10.0 \mathrm{~mm}$ for band length, 60.0 $\mathrm{mm}$ from the margin, and $16.0 \mathrm{~mm}$ of distance between tracks. They were spotted at a constant rate of $100 \mathrm{nl} / \mathrm{s}$ using nitrogen aspirator. The mobile phase used for plate development was toluene: ethyl acetate: acetic acid: methanol $(8: 2: 0.1: 0.2 \mathrm{v} / \mathrm{v} / \mathrm{v} / \mathrm{v})$. The development of TLC plate was carried out manually in the twin trough chamber with saturation duration of 30 minutes prior the development and $85.0 \mathrm{~mm}$ of migration distances. The plate was visualized at $254 \mathrm{~nm}$ and $366 \mathrm{~nm}$ in order to observe all compounds that can be seen under both wavelengths. Then, the densitometric analysis of all separated compounds also was carried out at 254 and $366 \mathrm{~nm}$ by CAMAG TLC Scanner 4. All assigned compounds in respective $R_{f}$ values in all samples for both wavelengths were scanned using tungsten and deuterium lamps at a constant scanning speed of $20 \mathrm{~mm} / \mathrm{s}$ with micro slit dimension of 10.0 $\mathrm{mm} \times 0.2 \mathrm{~mm}$, Macro. The scanning of all assigned compounds for both wavelengths was done on purpose for HPTLC-chemometric data extraction.

\subsection{Chemometric Analysis}

\subsubsection{Pre-Processing Data}


For FTIR datasets (1795 x 6 datasets), each measurement was baseline corrected in order to minimize the differences between spectra due to baseline shifts and normalized using Shimadzu IRsolution Version 1.40 (Shimadzu Corporation) software. The end region of spectra (399-539 $\left.\mathrm{cm}^{-1}\right)$ was cut-off due to the instrumental noise. However, no pre-processing was done on HPTLC datasets (18 x 6 datasets for $254 \mathrm{~nm}$; $14 \times 6$ datasets for $366 \mathrm{~nm})$ as this analysis was carried out on TLC silica gel plate which the baseline noise was not critical in the observation. All propolis samples were standardized into the same concentration prior the analysis $(30 \mathrm{mg} / \mathrm{mL})$ in order to ensure the concentration factor would not affect the clustering in chemometrics analysis. For FTIR numerical datasets, the file of .txt format was imported from Shimadzu IRsolution software and transferred into Microsoft Excel (rows: samples, columns: absorbance values for each wavelength). Meanwhile, the datasets of retention factor $\left(R_{f}\right)$ values and peak areas of all assigned compounds in all samples were extracted manually into Microsoft Excel 2007. All datasets were subjected to chemometrics analysis (PCA and HCA) which conducted with multivariate statistical analysis software, XLSTAT Pro 2014 (Addinsoft, Paris, France), an add-in software program for Microsoft Excel 2007.

\subsubsection{Principal Component Analysis (PCA)}

Principal Component Analysis is a technique that allows the identification of a group between variables, which reduce the dimensionality of data sets [20]. PCA also provides information on the most important parameters that explain the entire data sets by excluding the less significant parameters [21]. However, the PCs generated by PCA are sometimes not readily interpreted; thus it is advisable to rotate the PCs by varimax rotation to obtain new groups of variables called varimax factors (VFs) [22]. With the aim in obtaining the new groups of variables (varimax factors), varimax rotation was applied on the PCs with eigenvalues more than 1 are considered significant [23]. For loading plot, the higher the factor loading of that variable, the more the variable contributes to the variation accounted for the particular PC [24]. The combination of scores plot and loading plots were given out biplot scores.

\subsubsection{Hierarchical Cluster Analysis (HCA)}

Cluster analysis (CA) was defined as natural association of unlabeled data, which data 
samples were classified into clusters or classes in term of samples similarity within the classes and samples dissimilarity between classes defined by the entered variables without making earlier assumptions regarding the potential structure of the datasets [25-26]. There are two common approached that can be applied in cluster analysis, which hierarchical methods and non-hierarchical (k-mean clustering) methods [27]. While, hierarchical methods were divided into two sub methods which are agglomerative and divisive methods. Agglomerative method is many-to-one clustering concept, in which the samples that most similar to each other were start to combine together within the groups and repeatedly done until all samples under in one cluster. Meanwhile, divisive method applied reversely, one-to-many clustering concept in which all the samples start in same cluster and the samples that having dissimilarities separated apart into their own clusters. There are several different clustering methods and distance measures applied to link between clusters/objects in HCA which clustering method such as complete linkage (maximum distance), single linkage (minimum distance), average linkage (average distance), median linkage and Ward's method and distance measures such as Euclidean, Squared Euclidean, correlated, uncorrelated and etc. However, the most commonly used HCA method were Ward's method and Euclidean distance measures for dissimilarity [21, 26]. According to [26], horizontal lines in HCA dendograms was referred to the linkage between two objects/ clusters and vertical axis indicating to the height as a distance measure. The vertical line might represent to similarity or dissimilarity measures used in the analysis. In this work, hierarchical-agglomerative method was applied using Ward's method and Euclidean distance measures. The automatic truncation was checked prior analysis in order to let the hierarchical tree being cutting off for significant cluster, which formed in dotted line perpendicular to the hierarchical tree. The higher relative distances between the samples in the dendrogram tree indicate the dissimilarities between the samples increased.

\subsection{Antioxidant Activity}

\subsubsection{Qualitative HPTLC-DPPH Method}

DPPH reagent was used for post-chromatogram derivatization in order to observe the particular compounds that have antioxidant properties. The developed TLC plates as described in 2.4 were sprayed with $0.04 \%$ of methanolic DPPH solution according to method 
described in [28] with slightly modification. The TLC plates were incubated in the dark for 30 minutes and were then visualized under white light using CAMAG TLC Visualizer (DXA252 digital camera). The lower white light was used in order to thoroughly observe the antioxidant properties of all the bands of compounds.

\subsubsection{Quantitative DPPH Method}

The antioxidant activity, 2, 2-diphenyl-1-picrylhydrazyl (DPPH) free radical scavenging assay was performed according to [29] with slightly modification. Five milligram of the stock solutions of samples extracts and standards (controls) were prepared in $1 \mathrm{~mL}$ of DMSO. All samples and standards were made serial dilutions in 96-well micro litre plate from the stock solutions to get final concentration of $500,250,125,62.5,31.25,15.625$ and $7.8125 \mu \mathrm{g} / \mathrm{mL}$ in each respectively wells. Quercetin and Trolox standards were used as positive controls. The samples and standards then were mixed with $0.125 \mathrm{mM} \mathrm{DPPH}$ in methanol to produce a final DPPH concentration of $0.1 \mathrm{mM}$ in final volume of $250 \mu \mathrm{L}$ each well. The mixture of samples and DPPH solutions was allowed to incubate in the dark for 30 minutes at room temperature. The reaction was measured by absorbance at $517 \mathrm{~nm}$. The percentage of inhibition was calculated using the following formula.

Percentage of inhibition $=\left[1-\left(\mathrm{A}_{517 \mathrm{~nm} \text {, sample }} / \mathrm{A}_{517 \mathrm{~nm} \text {, control }}\right) \times 100\right]$

\subsubsection{Total Phenolic Content (TPC) Method}

The total phenolic contents were estimated by using Folin-Ciocalteau method in micro liter plate as described in [30] with some modification. The total phenolic contents in the extracts were expressed as Gallic Acid equivalents (GAE) which mg of gallic acid per $\mathrm{g}$ of samples extracts. The standard stock solution of gallic acid $(1 \mathrm{mg} / \mathrm{mL})$ were prepared and were made serial dilutions to produce the final concentration of $200,160,120,80$ and $40 \mu \mathrm{g} / \mathrm{mL}$ in micro tubes. For samples extracts, $60 \mu \mathrm{L}$ of stock solution of each samples extracts $(5 \mathrm{mg} / \mathrm{mL})$ were added to get $1 \mathrm{mg} / \mathrm{mL}$ of final concentration of samples extracts. The Gallic acid and samples extracts solutions were then make up to $100 \mu \mathrm{L}$ with methanol. Two hundred microliters of Folin-Ciocalteau (F-C) reagent were pipetted into all micro tubes and were vortexed thoroughly to complete the final volume of $300 \mu \mathrm{L}$ each micro tubes. For the last step of the reaction, $800 \mu \mathrm{L}$ of $7.5 \%$ sodium carbonate $\left(\mathrm{Na}_{2} \mathrm{CO}_{3}\right)$ were added into each micro tubes in 
order to complete the reaction and turn the solution in to blue colour (Note: sodium carbonate is excluded in final volume of micro tube). All micro tubes were then incubated at room temperature for 2 hours. This two hours incubation at room temperature are the critical step which allows homogenous colour development as well as giving low standard error of mean (s.e.m) between samples replicates. Two hundred microliters of Gallic acid standards and sample extracts were transferred into 96-well micro plate from assay tubes and the absorbance of each well was measured at $765 \mathrm{~nm}$. The total phenolic content of samples extracts were calculated using linear regression equation obtained from Gallic Acid Equivalent (GAE) calibration curve and following formula:

$\mathrm{C}=\mathrm{cV} / \mathrm{m}$

where C: total phenolic content (mg of GAE / g of samples extract), c: the concentration of Gallic acid (mg/mL) established from calibration curve, V: final volume of sample extract and m: the weight of samples extract.

\section{RESULTS AND DISCUSSION}

\subsection{Metabolite Fingerprinting by FTIR and HPTLC Coupled with Chemometric} Analysis

Metabolite fingerprinting is a rapid classification of sample with complex nature such as propolis. This analysis does not require of the identification of individual compounds, but to compare pattern or 'fingerprints' of metabolites that change in given treatment. In this study, quality of processed and unprocessed propolis samples in three extraction methods were evaluated from metabolite fingerprinting by FTIR and HPTLC coupled with chemometric analysis (PCA and HCA). Fig. 1 shows FTIR fingerprints (Fig. 1a) and chemometric analysis for all six samples (PCA-Fig.1b and HCA-Fig. 1c). In general, FTIR fingerprints of all six samples were observed to have similar fingerprints pattern but different in peak intensities. All six samples were observed to differentiate by peak intensity into respected extraction method. While in term of extraction method, processed and unprocessed samples were differed by peak intensity and absorbance values. PCA scores plot in Fig. 1(b) revealed that all six samples were clustered apart from each other indicates the significant different between 
clusters. Factor scores showed that P-M, UP-S and P-MS were classified into PC1 while UP-M, P-S and UP-MS were classified into PC2 (data not shown). From that data, only UP-S (PC1) and P-S (PC2) were having strong score (positive value more than 1). The factor loadings that given out the variables, which give strong correlation with the factor in PC1 were $1506 \mathrm{~cm}^{-1}, 1522-1524 \mathrm{~cm}^{-1}, 1533 \mathrm{~cm}^{-1}, 1537-1545 \mathrm{~cm}^{-1}$ and $1555-1560 \mathrm{~cm}^{-1}$. While the variables that give strong correlation with the factor in PC2 were $540-542 \mathrm{~cm}^{-1}, 548-760 \mathrm{~cm}^{-1}$, $878-885 \mathrm{~cm}^{-1}, 984-1099 \mathrm{~cm}^{-1}, 1155-1163 \mathrm{~cm}^{-1}, 1229-1292 \mathrm{~cm}^{-1}, 1366-1387 \mathrm{~cm}^{-1}, 1439-1468$ $\mathrm{cm}^{-1}$ and $2849-2974 \mathrm{~cm}^{-1}$.

The assignments of vibrational functional group of peaks in loading variables that responsible to the factor were attributed to $\mathrm{N}-\mathrm{H}$ bending in amides group of compounds. Meanwhile, for PC2: $540-542 \mathrm{~cm}^{-1}$ were assigned for C-C bending vibration, $548-760 \mathrm{~cm}^{-1}$ were assigned for out-of-plane bending with both substituted benzenes and bonded O-H group, 984-1099 $\mathrm{cm}^{-1}$ were the broad range for C-O stretching in alcohols group, $1155-1163 \mathrm{~cm}^{-1}$ and $1229-1292$ $\mathrm{cm}^{-1}$ were the assignments of C-O stretching of carbonyl band to an ester, 1366-1387 $\mathrm{cm}^{-1}$ was attributed to symmetrical bending vibration of $\mathrm{C}-\mathrm{H}$ bonds in methyl group $\left(\mathrm{CH}_{3}\right)$, 1439-1468 $\mathrm{cm}^{-1}$ was assigned for asymmetrical bending vibration of $\mathrm{C}-\mathrm{H}$ bonds in methyl group $\left(\mathrm{CH}_{3}\right)$, and 2849-2974 $\mathrm{cm}^{-1}$ was exist in doublet for the assignments of asymmetrical and symmetrical methylene stretching vibrations. This indicates that those variables were the main parameters contributed to strong factor score in both PCs. These compounds occur from sonication method for both processed and unprocessed samples. In additions, loading variables in PC1 which attributed from amide group, indicate strong presence of alkaloid group in unprocessed samples from sonication method. This finding corroborated with report by [8], which identify the presence of alkaloid in propolis samples.

However, in Fig. 1(c), HCA clearly showed that FTIR fingerprints of processed and unprocessed propolis samples in three extraction method were classified into three clusters which based on their method of extractions. In Fig. 1(c), Cluster II was clearly separated into another HCA tree, which indicates that by sonication, specific features of compound were extracted. This might be in agreement with PCA results, which both P-S and UP-S samples were strong factor in $\mathrm{PC} 2$ and $\mathrm{PC} 1$ respectively. From this finding, it revealed that 
FTIR-chemometric could be used as a tool for quality study based on extraction method.
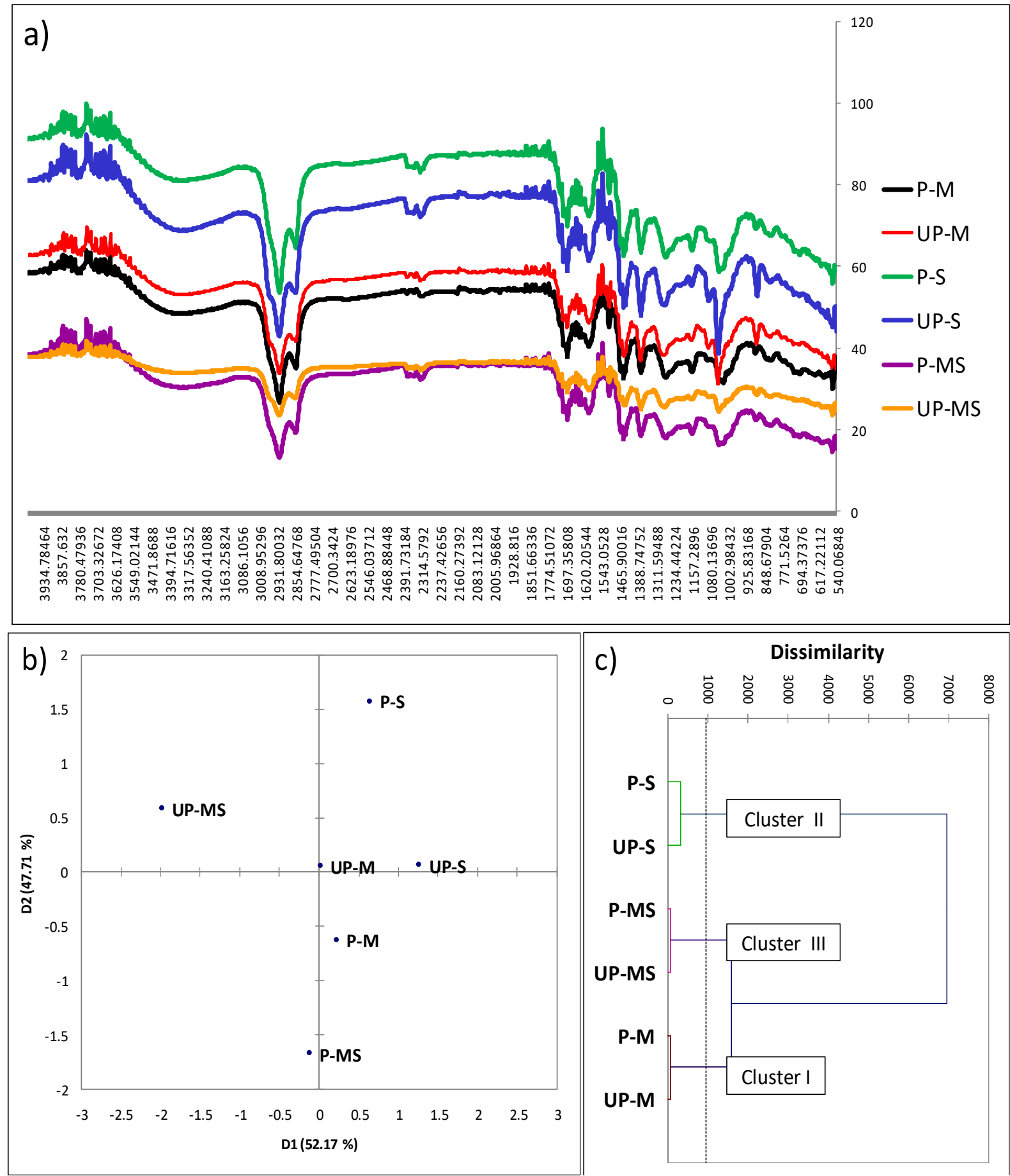

Fig.1. FTIR-chemometric of processed and unprocessed ethanolic extract of H. itama's propolis in three extraction methods. FTIR fingerprints for all samples (a), PCA score plot (b) and HCA dendrogram (c) of processed and unprocessed H. itama's propolis in three extractions method

Metabolite fingerprinting by HPTLC analysis and their chemometric analysis was carried out at two wavelengths, $254 \mathrm{~nm}$ (short wavelength) and $366 \mathrm{~nm}$ (long wavelength) in order to 
observe the differentiation between processed and unprocessed propolis samples. As for HPTLC profiling, the chemical compounds of unprocessed propolis samples in three different extraction methods were observed to be more intense in their HPTLC chromatogram at 254 and $366 \mathrm{~nm}$ (Fig. 2a and 2b). It was also found that there were compounds that absent in processed samples but occur in unprocessed samples and vice versa. This was clearly can be seen in HPTLC densitogram (Fig. 2c-f) in both wavelengths, which the peaks of compounds in unprocessed samples were more intense as compared to the processed one.

For data analysis of HPTLC-chemometric, all compounds at 254 and $366 \mathrm{~nm}$ were assigned according to their respective $R_{f}$ values. As results, there were 18 possible compounds found at $254 \mathrm{~nm}$ (Fig. 3) and 14 compounds at $366 \mathrm{~nm}$ (Fig. 4). The UV spectra comparison of those compounds shown in Fig. 3 and 4. The compounds were named based on their $R_{f}$ values. At $254 \mathrm{~nm}$ (Fig. 3), 10 compounds (0.07, 0.20, 0.31, 0.37, 0.45, 0.60, 064, 0.71, 0.78 and 0.91) were detected in both processed and unprocessed propolis. However, those compounds were suspected to have degraded based on their UV spectra in Fig. 3. In addition, four compounds were observed in non-heated (unprocessed) propolis in three extraction methods and were then absent after the propolis has been processed. Those four compounds were compounds at $R_{f}$ values of $0.12,0.49,0.52 \mathrm{a}$ and 0.86 (green circled in Fig. 3). In contrast, there were four additional compounds were observed to occur in heated propolis (processed). They are compounds at $R_{f}$ values of $0.33,0.40,0.52 \mathrm{~b}$ and 0.84 (red circled in Fig. 3). Similar case occurred in $366 \mathrm{~nm}$ (Fig. 4), which there was seven compounds $(0.12,0.21,0.31,0.49,0.65$, 0.78 and 0.90 ) were present in samples of processed and unprocessed. However, compounds at $R_{f}$ values of $0.65,0.78$ and 0.90 were degraded in processed samples. Compounds at $R_{f}$ values of $0.05,0.45$ and 0.74 were not seen processed samples. While, compounds at $R_{f}$ values of $0.07,0.38,0.41$ and 0.89 were observed in processed samples. As we compare UV spectra of compounds at 254 and $366 \mathrm{~nm}$, we found that there were 10 compounds were detectable at both wavelengths. Those 10 compounds were at $R_{f}$ values of $0.07,0.12$, $0.20-0.21,0.31,0.40-0.41,0.45,0.49,0.64-0.65,0.78$ and $0.90-0.91$. This analysis also revealed that there were eight specific compounds occur at $254 \mathrm{~nm}, R_{f}$ values of $0.33,0.37$, $0.52 \mathrm{a}, 0.52 \mathrm{~b}, 0.60,0.71,0.84$ and 0.86 while four specific compounds at $366 \mathrm{~nm}$, at $R_{f}$ values 
of $0.05,0.38,0.74$ and 0.89 .

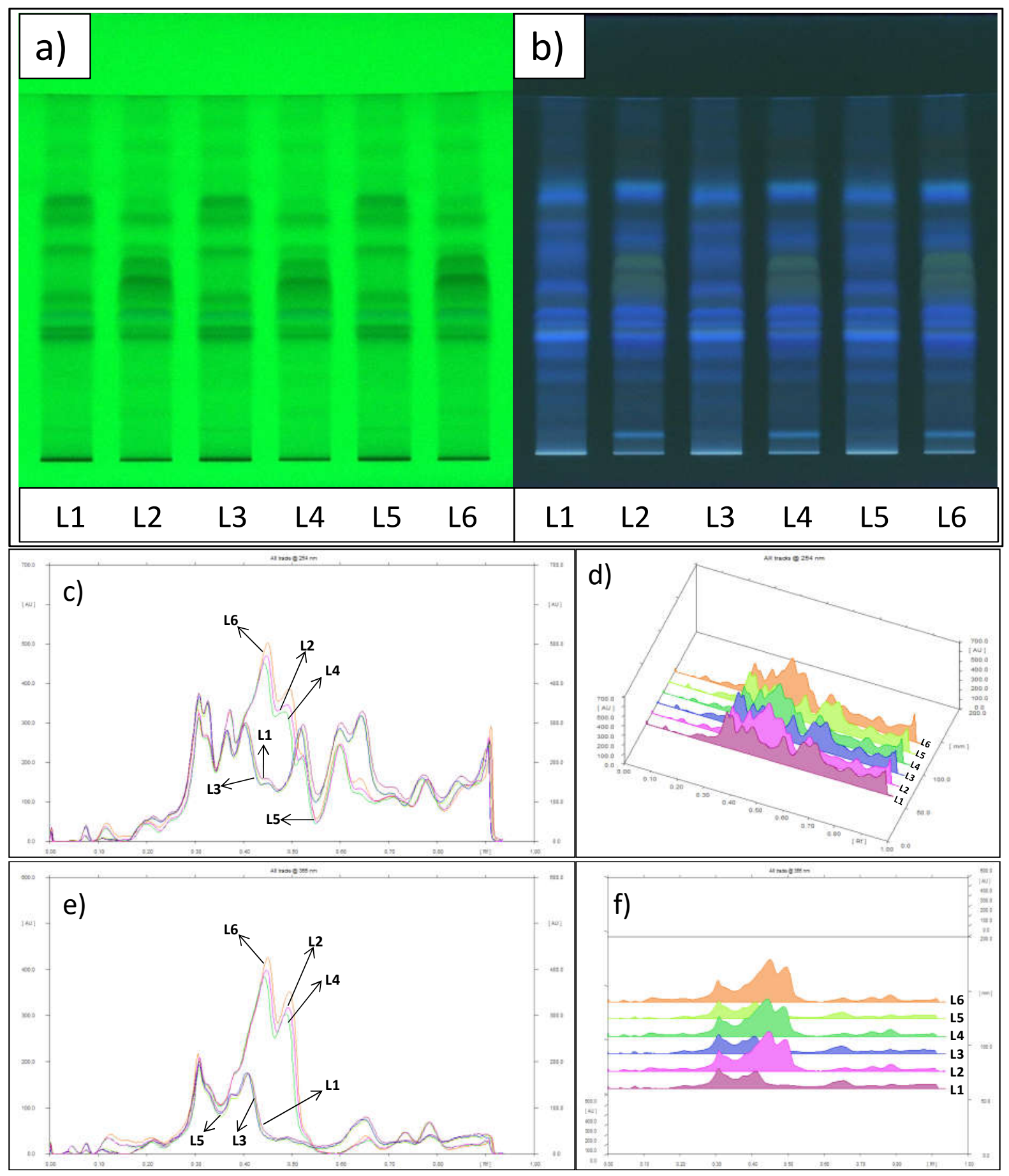

Fig.2. HPTLC profiling of processed and unprocessed ethanolic extract of $H$. itama's propolis in three extraction methods. HPTLC chromatograms viewed at $254 \mathrm{~nm}$ (a) and $366 \mathrm{~nm}$ (b). HPTLC densitogram under $254 \mathrm{~nm}-2 \mathrm{D}$ view (c), $254 \mathrm{~nm}-3 \mathrm{D}$ view (d), $366 \mathrm{~nm}-2 \mathrm{D}$ view (e) and 366 nm-3D view (f). L1: P-M; L2: UP-M; L3: P-S; L4: UP-S; L5: P-MS; L6: UP-MS 


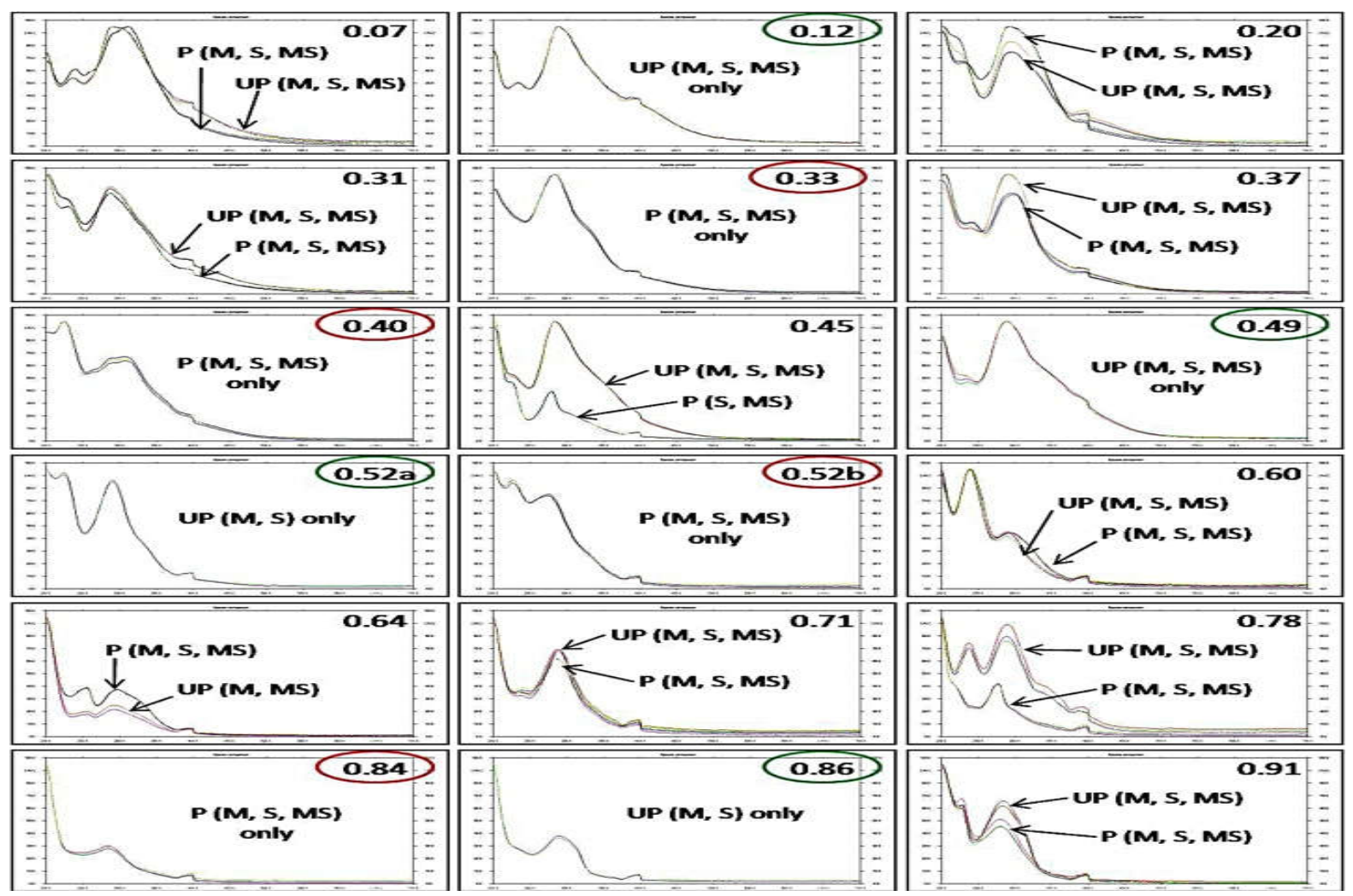

Fig.3. UV spectra comparison of all assigned peak of compounds in processed and unprocessed ethanolic extract of $H$. itama's propolis in three extraction methods by HPTLC analysis at $254 \mathrm{~nm}$

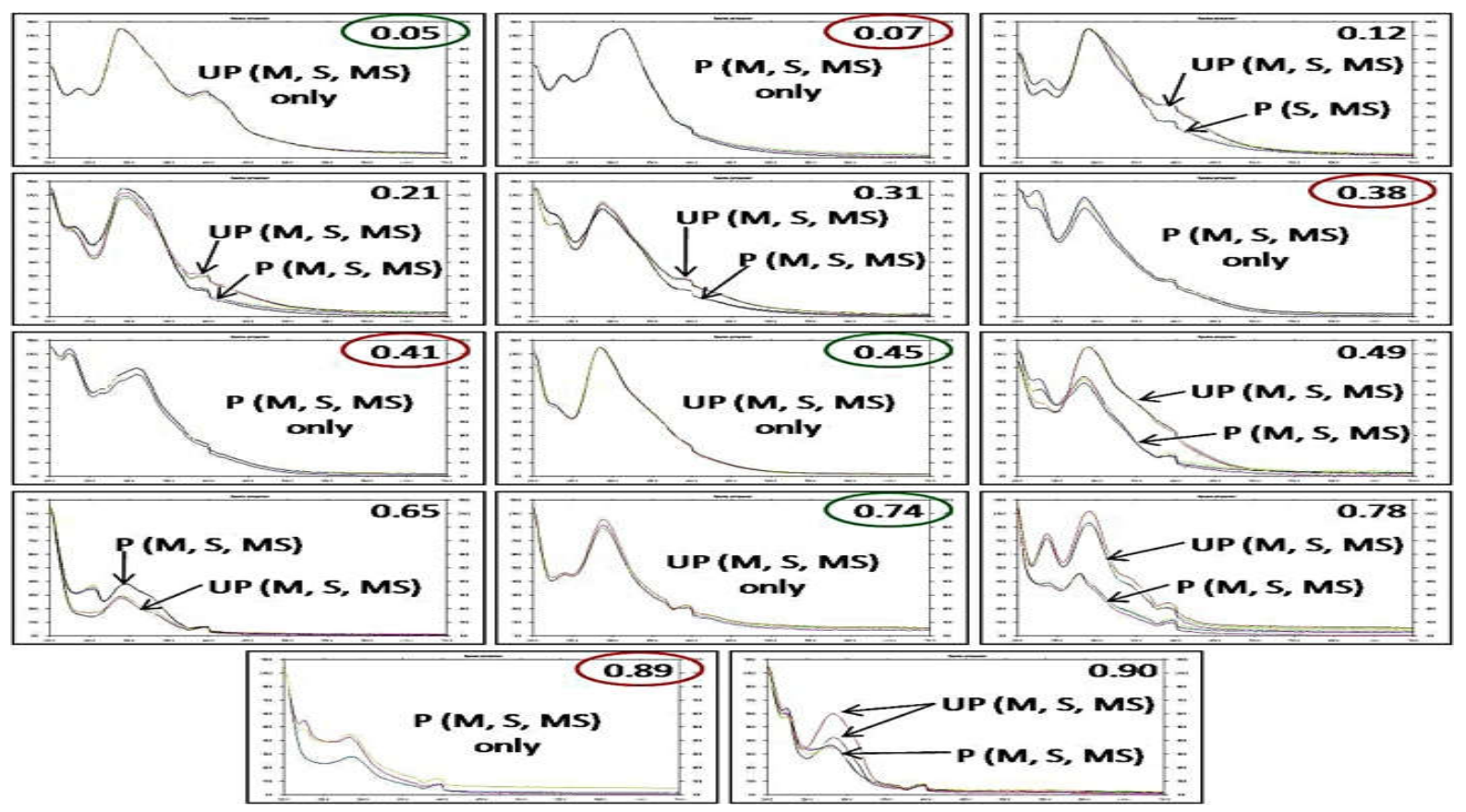

Fig.4. UV spectra comparison of all assigned peak of compounds in processed and unprocessed ethanolic extract of $H$. itama's propolis in three extraction methods by HPTLC analysis at $366 \mathrm{~nm}$ 
The complicated and confusing HPTLC retention factor numerical data at both 254 and 366 $\mathrm{nm}$ apparently could not be well summarized and concluded by human vision. In order to determine the similarity and dissimilarity, HPTLC datasets were then subjected to chemometric analysis (PCA and HCA). Fig. 5a and 5b show PCA scores plot for 254 and 366 $\mathrm{nm}$ respectively. In general, both PCA scores plot grouped in two clusters, respectively that separate unprocessed and processed samples apart. Factor scores of $254 \mathrm{~nm}$ (data not shown) grouped P-S, P-M, P-MS and UP-S into PC1 while factors in PC2 were UP-MS and UP-M. Factors of PC1 were observed located in lower right diagonal $\left(45^{\circ} \mathrm{C}\right)$, while factors of $\mathrm{PC} 2$ are located in upper left diagonal in PCA score plot (Fig. 5a). However, only P-M, P-S and P-MS are having positive values more than 1 in PC1 while UP-MS in PC2 which considered on having strong correlation with variables in the factor loadings. For $254 \mathrm{~nm}$, factor loadings given out the variables that have strong correlation (positive values) with positive factors (P-M, P-S and P-MS) in PC1 were compounds at $R_{f}$ values of 0.07, 0.20, 0.33, 0.40, 0.52b, 0.64, 0.78 and 0.84 (Fig. 5c). While UP-MS, positive factor in PC2 was strongly correlated to a variable in PC2 is compound at $R_{f}$ value 0.91 . Nevertheless, UP-MS, UP-M and UP-S were found to have directly correlation with variables that have negative values and do not correlate with positive factors in PC1 (Fig. 5c). As for $366 \mathrm{~nm}$ (Fig. 5b), UP-M, UP-S and P-MS were classified into PC1 while P-M, P-S and UP-MS in PC2 based on factor scores (data not shown). It clearly shows that positive factors in PC1 were UP-M and UP-S, while UP-MS is positive factor in PC2. Fig. 5d showed that these three samples were having strong correlation to variables with positive factor loadings in PC1, which compounds at $R_{f}$ values of $0.05,0.12,0.45,0.49,0.74$ and 0.78 . UP-MS (positive factor in PC2) also shows to have strong correlation with compound at $R_{f}$ values of 0.21 and 0.31 . Meanwhile, it was also found that P-MS, P-M and P-S (which have negative score and low positive values in PC1 and PC2 respectively) were strongly correlated to variables with negative factor loadings in PC1 (Fig. 5d). In brief, PCA gives distinct cluster regardless extraction method based on their specific compounds. 

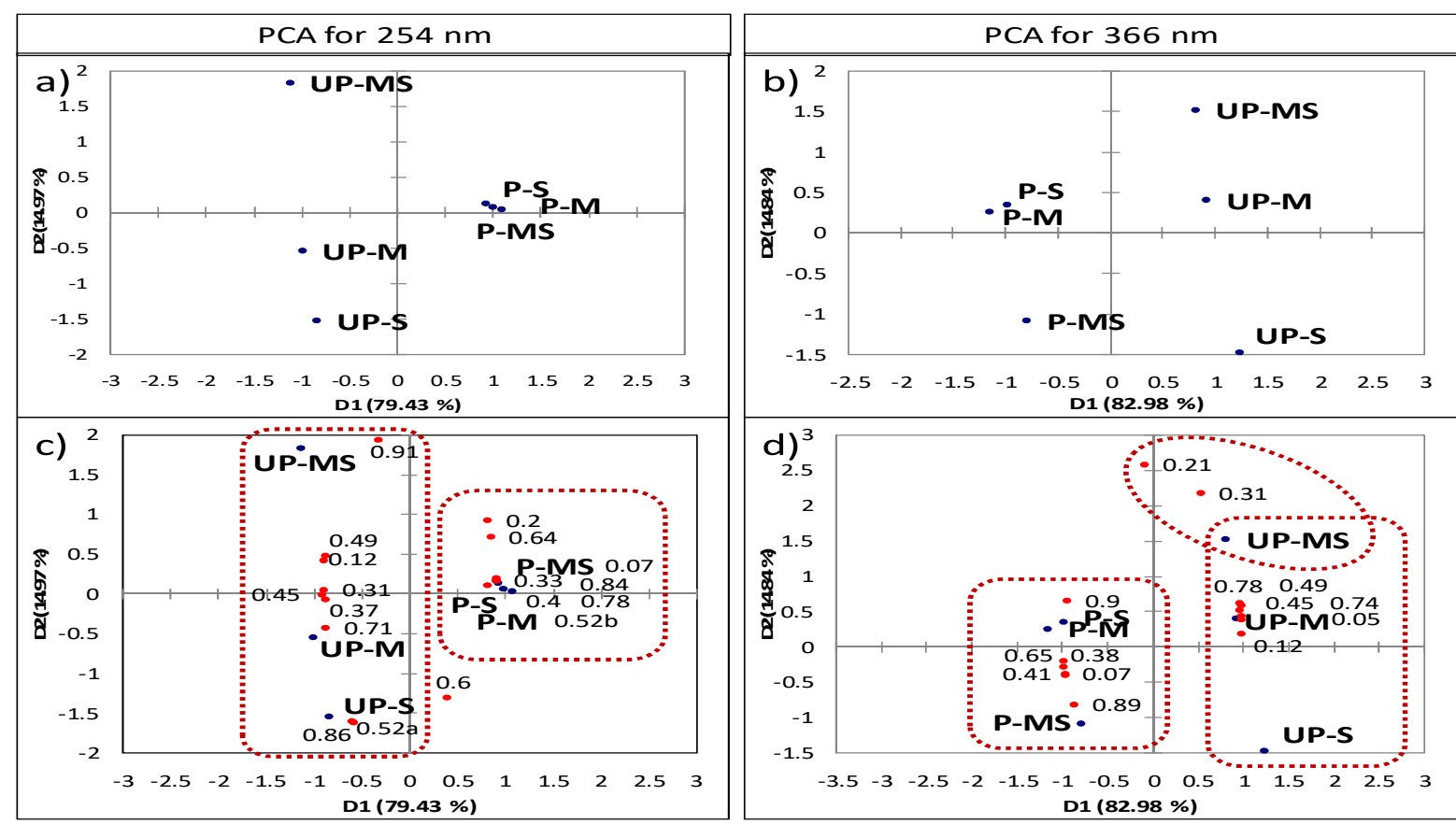

Fig.5. HPTLC-chemometric of processed and unprocessed ethanolic extract of H. itama's propolis in three extraction methods. PCA scores plot for HPTLC-254 nm (a) and HPTLC-366 nm (b). Biplot scores for HPTLC-254 nm (c) and HPTLC-366 nm (d)

Fig. 6 (a) and 6 (b) show that HPTLC-HCA dendrograms for both wavelengths, 254 and 366 $\mathrm{nm}$, respectively. Processed and unprocessed propolis samples were clearly divided into three clusters for both 254 and $366 \mathrm{~nm}$. Cluster 1 consists of processed propolis and unprocessed samples were divided into two cluster. Despite separated into two different clusters unprocessed propolis still show similar pattern for their profile plots (Fig. 6c and 6d). The pattern of profile plots revealed that compounds at $R_{f}$ values of $0.31,0.37,0.45,0.49,0.60$, 0.71 and 0.78 could be the identity for unprocessed propolis samples for $254 \mathrm{~nm}$. While compounds at $R_{f}$ values of $0.31,0.45,0.49$ and 0.78 could be the identity for that of $366 \mathrm{~nm}$. In addition, profile plot patterns of 254 and $366 \mathrm{~nm}$ also revealed that processed and unprocessed propolis samples possessed different pattern of compounds. Based on profile plot pattern of processed propolis samples, compounds at $R_{f}$ values of $0.33,0.40,0.52 \mathrm{~b}$ and 0.84 (might occur after heating process) could be the identity of compounds at $254 \mathrm{~nm}$, while at $366 \mathrm{~nm}$, compounds at $R_{f}$ values of $0.38,0.41$ and 0.89 could be the identity for processed propolis samples regardless extraction methods. HPTLC-chemometric in this work revealed that, the quality of propolis does affected by the heating process and not by the extraction method. 


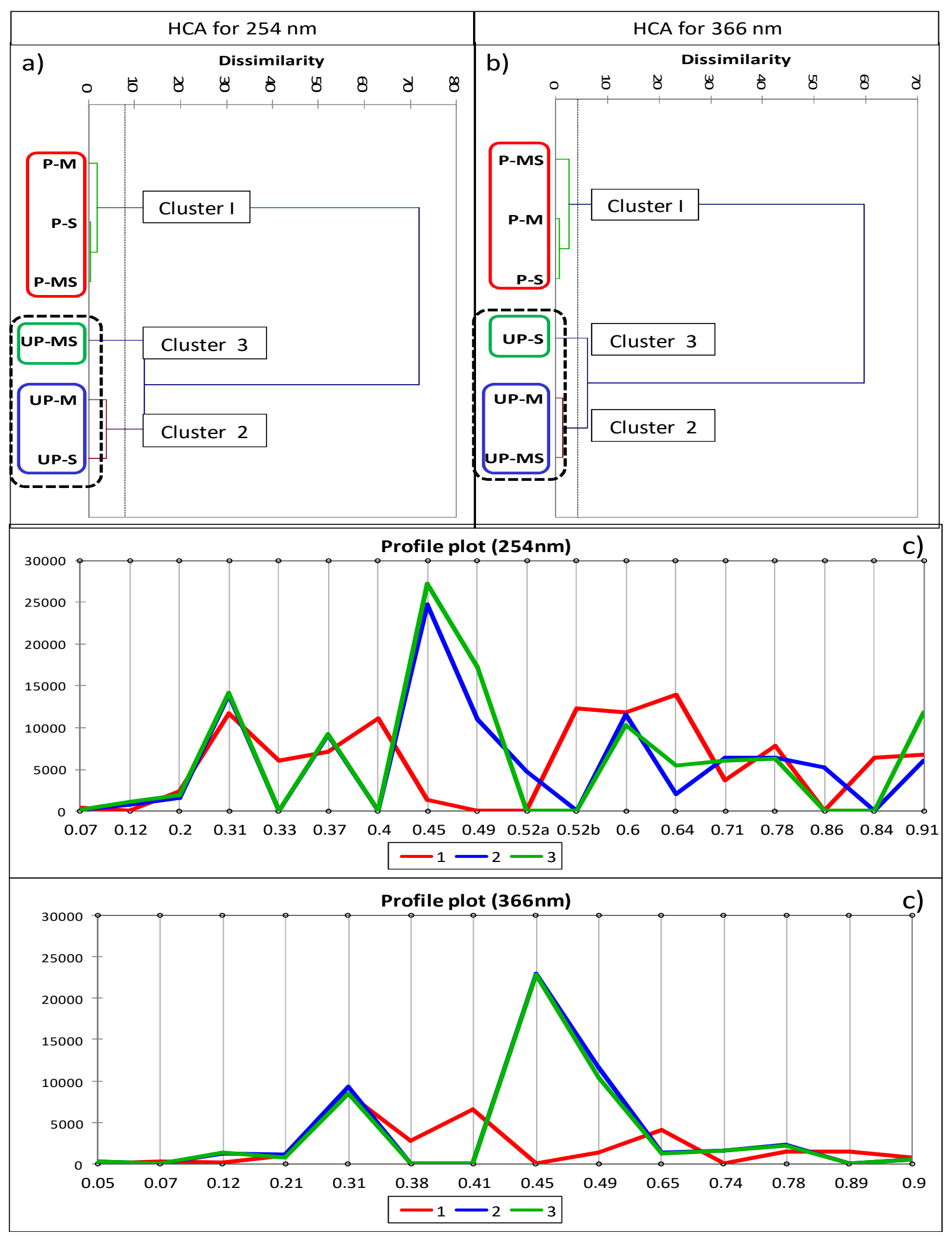

Fig.6. HPTLC-chemometric of processed and unprocessed ethanolic extract of H. itama's propolis in three extraction methods. HCA dendrograms for $254 \mathrm{~nm}$ (a) and $366 \mathrm{~nm}$ (b).

Profile plot of compounds variables classified by HCA dendrograms for 254 and $366 \mathrm{~nm}$ in three clusters HCA 


\subsection{Antioxidant Activity}

Processed and unprocessed propolis for three extraction methods were also tested on antioxidant activity by DPPH and Total Phenolic Content (TPC) methods. Fig. 7 (a) and 7(c) shows the result of quantitative DPPH assay. Unprocessed propolis possessed significantly higher antioxidant activity as compared to the processed propolis. This result was in agreement with qualitative HPTLC-DPPH assay as shown in Fig. 7 (b). Unprocessed propolis (L2, L4 and L6) was observed to have more compounds that scavenged the DPPH free radicals better than that of processed propolis (L1, L3 and L5). In term of method of extraction, those three methods were significantly different to each other. Based on $\mathrm{IC}_{50}$ values, antioxidant activity of unprocessed propolis ranked in order of UP-MS $(62.1 \mu \mathrm{g} / \mathrm{mL})>$ UP-M $(73.9 \mu \mathrm{g} / \mathrm{mL})>$ UP-S $(85.7 \mu \mathrm{g} / \mathrm{mL})$. While for processed propolis samples, maceration-sonication method ( $\mathrm{IC}_{50}$ value: P-MS; $172.3 \mu \mathrm{g} / \mathrm{mL}$ ) was significant different to both maceration and sonication methods, but both maceration ( $\mathrm{IC}_{50}$ value: $\mathrm{P}-\mathrm{M} ; 154.8 \mu \mathrm{g} / \mathrm{mL}$ ) and sonication ( $\mathrm{IC}_{50}$ value: $\mathrm{P}-\mathrm{M} ; 157.1 \mu \mathrm{g} / \mathrm{mL}$ ) methods were no significantly different from each other. It was found that maceration-sonication method give the highest antioxidant activity in unprocessed samples but the lowest activity in processed samples. This indicates that extraction method is not a reliable method for quality control parameter for propolis raw material. All six samples were found significantly different with positive controls used; quercetin ( $\mathrm{IC}_{50}$ value: $5.2 \mu \mathrm{g} / \mathrm{mL}$ ) and Trolox ( $\mathrm{IC}_{50}$ value: $6.0 \mu \mathrm{g} / \mathrm{mL}$ ). In summary of DPPH antioxidant activity, HPTLC-DPPH analysis shows that some of the antioxidant compounds were degraded or loss their ability after being heated in high temperature.

\subsection{Total Phenolic Content (TPC)}

Fig. 7(d) and 7(e) show that total phenolic content in processed and unprocessed samples and Gallic acid equivalent calibration curve, respectively. Fig. 7(d) revealed that unprocessed propolis consisted higher content of phenolic than processed propolis. The unprocessed propolis samples significantly higher than processed one in maceration (UP-M: $21.23 \mathrm{mg} / \mathrm{g}$; P-M: $17.47 \mathrm{mg} / \mathrm{g}$ ) and maceration-sonication (UP-MS: $21.94 \mathrm{mg} / \mathrm{g} ;$ P-MS: $18.17 \mathrm{mg} / \mathrm{g}$ ) methods. Sonication method however gives no significantly different in phenolic content for unprocessed and processed propolis samples (UP-S: $20.19 \mathrm{mg} / \mathrm{g}$; P-S: $17.89 \mathrm{mg} / \mathrm{g}$ ). In 
additions, there is no significantly different of phenolic content in extracts derived from three extraction methods of processed and unprocessed propolis. These findings revealed that, unprocessed propolis give out higher amount of phenolic compared to processed one if they were extracted using maceration and maceration-sonication methods and we could use any one of these two extraction methods as they were no significant different to each other. The phenolic content was proved are affected by processing method of propolis.

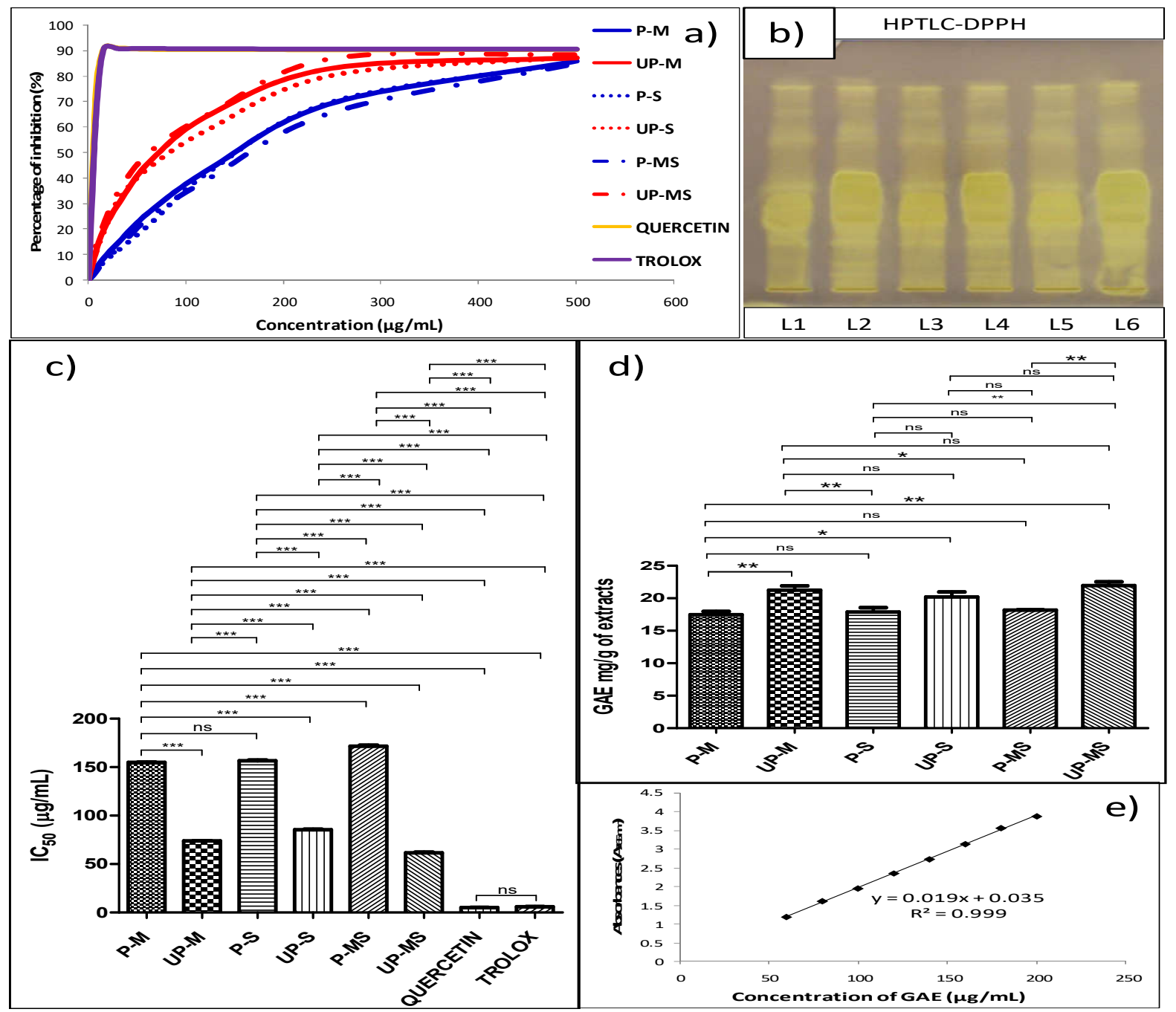

Fig.7. Antioxidant activity of processed and unprocessed ethanolic extract of H. itama's propolis in three extraction methods. Percentages of inhibition graph for 96-well plate DPPH method (a) and HPTLC-DPPH method (b). L1: P-M; L2: UP-M; L3: P-S; L4: UP-S; L5:

P-MS; L6: UP-MS. The graph for $\mathrm{IC}_{50}$ values of quantitative DPPH method (c). Total phenolic content (d) and calibration curve of Gallic Acid Equivalents. Means within samples indicate significantly different values $(* * *: \mathrm{p}<0.0001 ; * *$ : $\mathrm{p}<0.01 ; *$ : $\mathrm{p}<0.05$; ns: no significant) 


\subsection{Summary}

One of the oldest forms of processing and preserving food or raw material is heating and drying. The aim is to extend the shelf life of certain material, minimize packaging and storaging (as the case for propolis in this study) and reduce shipping weights [9]. The effect of processing using the high temperatures can have negative effects on flavour and nutritional content of the final products [10-11]. Heating is accountable for the oxidation, thermal degradation and leaching of bioactive from fresh raw material. Different heating conditions have different effects on the chemical profiles and antioxidant properties of plant material [12]. In this study, FTIR and HPTLC analyses were used to evaluate the effect of heating on propolis raw material. FTIR is a spectroscopic technique that is commonly used for the fingerprinting of different natural matrices, especially for quality control and for rapid qualitative evaluation of sample preparation [13]. In this study, FTIR analysis is able to significantly separate all samples. Heating process together with different type of extraction method does have an effect on the overall metabolite profile of propolis sample. Due to its simplicity, low cost and ability to simultaneously analyze up to 20 samples under same condition, HPTLC was employed to evaluate the effect on heating process on chemical profile of propolis extracts. TLC fingerprinting was widely used in assessment of the authenticity of different type of propolis [14-16]. The system was optimized to separate secondary metabolites. HPTLC chromatogram presented in this study show different chemical pattern and chemometric analysis by PCA and HCA confirm this observation. It clearly shows that heating largely affect the chemical composition especially secondary metabolites as shown from HPTLC analysis. This result was further verified by the evaluation of antioxidant activity, where the activity was markedly decreased in processed propolis. Qualitative analysis by HPTLC-DPPH shows substantial numbers of compounds in processed propolis was inactive against DPPH. The antioxidant capacity of heated samples decreased as a consequence of antioxidant compounds being relatively unstable at higher temperature. The decrease may also link to the formation of compounds having pro-oxidant action [17]. The effect of heating on total phenolic content was not as dramatic as antioxidant capacity eventhough it decreasing. Phenolic compounds are the most active derivatives in plant 
products [18]. There are reports stated that heating plant material at $150^{\circ} \mathrm{C}$ for 30 minutes decreased the total phenolic content. But, heating also able to liberated phenolic compounds by cleavage of esterified and glycosylated bond or by the formation of Maillard reaction products and produced free phenolic [19]. This might be the reason on less degradation of total phenolic compounds.

\section{CONCLUSION}

From this finding, it revealed that FTIR-chemometric could be used as a tool for quality study based on extraction method. Meanwhile, HPTLC-chemometric in this work could be used as quality tools for processing method of propolis, as processed and unprocessed propolis samples given out different pattern regardless extraction method. As overall conclusion for both DPPH method and TPC for antioxidant activity, phenolic compounds could be assigned as factors that contribute to antioxidant activity of propolis for processing method. Both DPPH and TPC methods were could be used as quality and efficacy parameters for quality control of propolis. However, only maceration method that observed to be reliable method in quality control purposes from these two antioxidant methods. In this work, metabolite fingerprinting by FTIR and HPTLC coupled with chemometric analysis and antioxidant activity were reliable for quality control for product development of propolis raw materials.

\section{ACKNOWLEDGEMENTS}

The authors are very thankful to the YMR International Marketing Sdn Bhd for providing the samples. This study was supported by Research Acculturation Collaborative Effort (RACE) Grant Scheme (No. RACE/F1/SG2/UniSZA/1: RR109) and Knowledge Transfer Grant Scheme (KTP) (No. F1-FS/8 (UNISZA-15: M). Both grant are from Ministry of Higher Education of Malaysia.

\section{REFERENCES}

[1] Pietta P G, Gardana C, Pietta A M. Analytical methods for quality control of propolis. Fitoterapia, 2002, 73(1):7-20 
[2] Ibrahim N, Zakaria A J, Ismail Z, Mohd K S. Antibacterial and phenolic content of propolis produced by two Malaysian Stingless Bees, Heterotrigona itama and Geniotrigona thoracica. International Journal of Pharmacognosy and Phytochemical Research, 2016, $8(1): 156-161$

[3] Alencar S M, Oldoni T L C, Castro M L, Cabral I S R, Costa-Neto C M, Cury J A, Rosalen P L, Ikegaki M. Chemical composition and biological activity of a new type of Brazilian propolis: Red propolis. Journal of Ethnopharmacology, 2007, 113(2):278-283

[4] a) Kumazawa S, Hamasaka T, Nakayama T. Antioxidant activity of propolis of various geographic origins. Food Chemistry, 2004, 84(3):329-339; b) Lanez T. et al. Evaluation of Antioxidant Capacity of Propolis Collected in Various Areas of Algeria Using Electrochemical Techniques, Int. J. Electrochem. Sci., 2015, (10) 9641 - 9651; c) Lanez T. et al. In vitro Evaluation of Antioxidant Capacity of Algerian Propolis by spectrophotometrical and Electrochemical Assays, Int. J. Pharmacol., 2011, 7 (1) 113-118; d) Lanez T. et al. Total polyphenol contents, radical scavenging and cyclic voltammetry of Algerian propolis, Int $\mathrm{J}$ Pharm Pharm Sci, 2014, 6 (1), 395-400.

[5] Kujumgiev A, Tsvetkova I, Serkedjieva Y, Bankova V, Christov R, Popov S. Antibacterial, antifungal and antiviral activity of propolis of different geographic origin. Journal of Ethnopharmacology, 1999, 64(3):235-240

[6] Ramos A F, Miranda J D. Propolis: a review of its anti-inflammatory and healing actions. Journal of Venomous Animals and Toxins Including Tropical Diseases, 2007, 13(4):697-710 [7] Xuan H, Li Z, Yan H, Sang Q, Wang K, He Q, Wang Y, Hu F. Antitumor activity of Chinese propolis in human breast cancer MCF-7 and MDA-MB-231 cells. Evidence-Based Complementary and Alternative Medicine, 2014, 2014:1-12

[8] Fatima J, Majid B, Nima B. Antimicrobial activity and chemical screening of propolis extracts. American Journal of Life Sciences, 2014, 2(2):72-75

[9] Izli G. Total phenolics, antioxidant capacity, colour and drying characteristics of date fruit dried with different methods. Food Science and Technology , 2017, 37(1):139-147

[10] Sharma K, Ko E Y, Assefa A D, Ha S, Nile S H, Lee E T, Park S W. Temperature-dependent studies on the total phenolics, flavonoids, antioxidant activities, and 
sugar content in six onion varieties. Journal of Food and Drug Analysis, 2015, 23(2):243-252. [11] Turkmen N, Sari F, Velioglu Y S. The effect of cooking methods on total phenolics and antioxidant activity of selected green vegetables. Food Chemistry, 2005, 93(4):713-718

[12] Makris D P, Rossiter J T. Domestic processing of onion bulbs (Allium cepa) and asparagus spears (Asparagus officinalis): Effect on flavonol content and antioxidant status. Journal of Agricultural and Food Chemistry, 2001, 49(7):3216-3222

[13] Wolfender J L, Marti G, Thomas A, Bertrand S. Current approaches and challenges for the metabolite profiling of complex natural extracts. Journal of Chromatography A, 2015, $1382: 136-164$

[14] Milojković Opsenica D, Ristivojević P, Trifković J, Vovk I, Lušić D, Tešić Ž. TLC fingerprinting and pattern recognition methods in the assessment of authenticity of poplar-type propolis. Journal of Chromatographic Science, 2016, 54(7):1077-1083

[15] Azemin A, Dharmaraj S, Hamdan M R, Mat N, Ismail Z, Khamsah S M. Discriminating ficus deltoidea var. bornensis from different localities by HPTLC and FTIR fingerprinting. Journal of Applied Pharmaceutical Science, 2014, 4(11):69-75

[16] Mohd K S, Azemin A, Hamil M S R, Bakar A R A, Dharmaraj S, Hamdan M R, Mohamad H, Mat N, Ismail N. Application of high performance thin layer chromatography and fourier transform infrared profiling coupled with chemometrics for the differentiation of the varieties of Ficus deltoidea Jack. Asian Journal of Pharmaceutical and Clinical Research, 2014, 7(5):110-116

[17] Nicoli M C, Anese M, Parpinel M. Influence of processing on the antioxidant properties of fruit and vegetables. Trends in Food Science and Technology, 1999, 10(3):94-100

[18] Vega-Gálvez A, Ah-Hen K, Chacana M, Vergara J, Martínez-Monzó J, García-Segovia P, Lemus-Mondaca R, Di Scala K. Effect of temperature and air velocity on drying kinetics, antioxidant capacity, total phenolic content, colour, texture and microstructure of apple (var. Granny Smith) slices. Food Chemistry, 2012, 132(1):51-59

[19] Maillard M N, Soum M H, Boivin P, Berset C. Antioxidant activity of barley and malt: relationship with phenolic content. LWT-Food Science and Technology, 1996, 29(3):238-244 [20] Martinez W. L., Martinez A. R., Martinez A., Solka J., Exploratory data analysis with 
MATLAB. Florida: CRC Press, 2010

[21] Singh K P, Malik A, Sinha S. Water quality assessment and apportionment of pollution sources of Gomti River (India) using multivariate statistical techniques-A case study. Analytica Chimica Acta, 2005, 538(1):355-374

[22] Retnam A, Zakaria M P, Juahir H, Aris A Z, Zali M A, Kasim M F. Chemometric techniques in distribution, characterization and source apportionment of polycyclic aromatic hydrocarbons (PAHS) in aquaculture sediments in Malaysia. Marine Pollution Bulletin, 2013, 69(1):55-66

[23] Juahir H, Zain S M, Yusoff M K, Hanidza T T, Armi A M, Toriman M E, Mokhtar M. Spatial water quality assessment of Langat River Basin (Malaysia) using environmetric techniques. Environmental Monitoring and Assessment, 2011, 173(1-4):625-641

[24] Saidan N H, Hamil M S, Memon A H, Abdelbari M M, Hamdan M R, Mohd K S, Majid A M, Ismail Z. Selected metabolites profiling of Orthosiphon stamineus Benth leaves extracts combined with chemometrics analysis and correlation with biological activities. BMC Complementary and Alternative Medicine, 2015, 15(1):1-12

[25] Gazzaz N M, Yusoff M K, Ramli M F, Aris A Z, Juahir H. Characterization of spatial patterns in river water quality using chemometric pattern recognition techniques. Marine Pollution Bulletin, 2012, 64(4):688-698

[26] Templ M, Filzmoser P, Reimann C. Cluster analysis applied to regional geochemical data: Problems and possibilities. Applied Geochemistry, 2008, 23(8):2198-2213

[27] Dominick D, Juahir H, Latif M T, Zain S M, Aris A Z. Spatial assessment of air quality patterns in Malaysia using multivariate analysis. Atmospheric Environment, 2012, 60:172-181 [28] Yrjönen T, Peiwu L, Summanen J, Hopia A, Vuorela H. Free radical-scavenging activity of phenolics by reversed-phase TLC. Journal of the American Oil Chemists' Society, 2003, $80(1): 9-14$

[29] Jo W S, Yang K M, Park H S, Kim G Y, Nam B H, Jeong M H, Choi Y J. Effect of microalgal extracts of Tetraselmis suecica against UVB-induced photoaging in human skin fibroblasts. Toxicological Research., 2012, 28(4):241-248

[30] Ainsworth A E, Gillespie K M. Estimation of total phenolic content and other oxidation 
substrates in plant tissues using Folin-Ciocalteu reagent. Nature Protocols, 2007, 2(4):875-877

\section{How to cite this article:}

Azemin A, Md-Zin NB, Mohd-Rodi MM, Kim-Chee AS, Zakaria, AJ, Mohd, KS. Assessing the quality of processed and unprocessed stingless bee's propolis using metabolite fingerprinting and antioxidant activity. J. Fundam. Appl. Sci., 2017, 9(2S), 637-660. 